\title{
Calidad de vida relacionada con la salud en pacientes con enfermedad inflamatoria intestinal
}

\section{Quality of life in patients with inflammatory bowel diseases}

\author{
R. López Cortés ${ }^{1}$, B. Marín Fernández², C. Hueso Montoro', P. Escalada Hernández², \\ A.C. Sanz Aznarez ${ }^{3}$, C. Rodríguez Gutiérrez
}

http: //dx.doi.org/10.4321/S1137-6627/2016000100014

\section{RESUMEN}

Fundamento. Determinar la calidad de vida relacionada con la salud en pacientes con enfermedad de Crohn y colitis ulcerosa, así como evaluar las diferencias en función del sexo, tipo de enfermedad y otras variables sociodemográficas.

Método. Estudio observacional, descriptivo y transversal en 100 pacientes atendidos en el servicio de Digestivo del Complejo Hospitalario de Navarra. Se empleó un cuestionario con variables sociodemográficas y clínicas, además del Inflammatory Bowel Disease Questionnaire-32, en su versión adaptada al castellano, para medir la calidad de vida.

Resultados. La media de la puntuación total del cuestionario de calidad vida es de 166 puntos (D.T.=40,06) sobre un máximo de 232. Se han hallado diferencias estadísticamente significativas en función del tipo de enfermedad $(\mathrm{p}=0,005)$ y el sexo $(\mathrm{p}=0,001)$.

Conclusiones. Las personas con enfermedad de Crohn o sexo femenino perciben peor calidad de vida relacionada con la salud respecto a pacientes con colitis ulcerosa u hombres.

Palabras clave. Enfermedad inflamatoria intestinal. Colitis ulcerosa. Enfermedad de Crohn. Calidad de vida.

\begin{abstract}
Background. To determine the health-related quality of life in patients with Crohn disease and ulcerative colitis, as well as to evaluate differences according to sex, type of disease and other sociodemographic variables.
\end{abstract}

Method. Cross sectional study of 100 outpatients in the Digestive Service of the Navarre Hospital Complex. A questionnaire with sociodemographic and clinical variables was used, as well as a version of the Inflammatory Bowel Disease Questionnaire-32 adapted to Spanish, in order to measure quality of life.

Results. The average score of the questionnaire on quality of life was 166 points (D.T.=40.06) out of a maximum of 232 . Statistically significant differences were found according to type of disease $(p=0.005)$ and $\operatorname{sex}(\mathrm{p}=0.001)$.

Conclusions. People with Crohn disease or females perceive a worse quality of life related to health in comparison to patients with ulcerative colitis or men.

Keywords: Inflammatory bowel disease. Ulcerative colitis. Crohn disease. Quality of life.
1. Departamento de Ciencias de la Salud. Universidad de Granada.

2. Facultad Ciencias de la Salud. Universidad Pública de Navarra. Pamplona.

3. Complejo Hospitalario de Navarra. Pamplona.

Recepción: 19-10-2015

Aceptación provisional: 10-12-2015

Aceptación definitiva: 11-01-2016

\section{Correspondencia:}

Blanca Marín Fernández

Departamento de Ciencias de la Salud Universidad Pública de Navarra Avenida de Barañain S/N 31008 Pamplona.

E-mail: blanca.marin@unavarra.es 


\section{INTRODUCCIÓN}

El término de enfermedades inflamatorias intestinales (EII) engloba una serie enfermedades crónicas del tracto gastrointestinal caracterizadas porque cursan con episodios de recidivas (brotes) que se alteran con periodos de remisión de la enfermedad. Las más comunes son la enfermedad de Crohn (EC) y la Colitis ulcerosa (CU) y se manifiestan con la aparición de síntomas intestinales como diarrea, heces con sangre y dolor abdominal. También pueden presentar síntomas extraintestinales tales como fatiga, pérdida de peso y dolor en las articulaciones ${ }^{1}$.

Este tipo de patologías son enfermedades crónicas que causan una gran afectación de la salud, que se evidencia en forma de cambios en las actitudes y conductas a nivel físico, emocional y social. Por ello, conseguir una mejor percepción de la calidad de vida es muy beneficioso y un punto clave de las mismas ${ }^{2}$.

La calidad de vida relacionada con la salud (CVRS) es una importante medida de percepción de la enfermedad por parte del paciente. Se entiende por CVRS aquella cualidad vital integrada por elementos físicos, sociales y psicológicos de la vida de los pacientes, determinados e influidos por las experiencias, creencias, expectativas del individuo en situación de salud y modificadas por el padecimiento de una enfermedad $^{3}$. La evaluación de una manera cuantitativa y fiable de la CVRS juega un importante papel no solo en la investigación aplicada en salud, sino también en el manejo terapéutico del paciente.

En esta línea, el Inflammatory Bowel Disease Questionnaire (IBDQ), desarrollado por Guyatt y col en $1989^{4}$ es el instrumento de medición de la calidad de vida más ampliamente usado en pacientes con EC y CU. El IBDQ en su versión de 32 preguntas (IBDQ-32) ha demostrado tener validez y fiabilidad adecuada, incluso en los países con diferentes lenguas, culturas y formas de vida. Por lo tanto, se recomienda la utilización de este instrumento en la investigación médica o evaluaciones de la CVRS en pacientes con $\mathrm{EI}^{5}$.
En algunos estudios realizados sobre CVRS en pacientes con EII, se ha demostrado que presentan una calidad significativamente menor que la población en general. Asimismo, la EII ha demostrado tener un impacto negativo en la salud relacionado con la CVRS especialmente cuando la enfermedad está activa ${ }^{6}$. En esta línea, otro estudio apunta que existe una relación inversa entre calidad de vida y actividad en $\mathrm{EII}^{3}$.

El objetivo de este trabajo fue analizar la CVRS de personas con EII, así como evaluar las diferencias en función del sexo, el tipo de enfermedad y otras variables sociodemográficas.

\section{MATERIAL Y MÉTODOS}

Se llevó a cabo un estudio observacional, descriptivo y transversal, entre los meses de septiembre y diciembre de 2014, en el que participaron 100 pacientes tratados en el servicio de Digestivo del Complejo Hospitalario de Navarra. El cálculo del tamaño muestral se estimó en 90 sujetos, considerado un error tipo I del $5 \%$, un error tipo II del $20 \%$ (potencia $80 \%$ ) y una diferencia mínima a detectar de calidad de vida entre los grupos de 25 puntos con una DE de 40 puntos $(\Delta=0,6)$. El reclutamiento de participantes fue realizado por el personal médico del servicio de Digestivo sin que tomara parte ningún miembro del equipo investigador, para evitar posibles sesgos, aplicando un criterio aleatorio según el orden de atención en la consulta. Así, finalizada la consulta, se le explicaba al paciente los objetivos del estudio y se le invitaba a participar. A continuación firmaban el consentimiento y pasaban a realizar una entrevista con un miembro del equipo investigador. Todos los pacientes invitados aceptaron participar en el estudio.

Los datos fueron obtenidos mediante una entrevista personal con cada participante, confirmando los datos clínicos con el médico de referencia y con la historia clínica del paciente. Para asegurar una recogida de datos sistemática, se diseñó un cuaderno de recogida de datos que fue utilizado por todos los miembros del equipo investigador. 
Las variables sociodemográficas y clínicas analizadas fueron: edad, sexo, nivel de estudios, situación laboral, estado civil, edad al diagnóstico de la EII, antecedentes familiares de EII, hábito tabáquico, presencia de otras enfermedades crónicas, grado de discapacidad y/o incapacidad reconocida oficialmente

Para la medición de la calidad de vida, se utilizó el cuestionario específico Inflammatory Bowel Disease Questionnaire de 32 ítems (IBDQ-32) en su versión adaptada y validada al castellano ${ }^{2}$, que agrupa la información en 4 dimensiones (síntomas intestinales y sistémicos, función emocional y social). Cada ítem se valora mediante una escala de Likert de 7 puntos de manera que el rango de la puntuación total del IBDQ-32 está entre 32 y 224, siendo las puntuaciones más altas indicativas de una mejor calidad de vida. Esta versión ha demostrado ser válida y discriminativa para ser la medida de la CVRS tanto en pacientes con enfermedad de Crohn como con colitis ulcerosa al poseer excelentes propiedades psicométricas, en términos de fiabilidad, reproducibilidad y sensibilidad a los cambios ${ }^{2,7}$.

Para la realización de este estudio se obtuvieron los permisos pertinentes de la dirección del centro y la evaluación positiva por parte del Comité Ético de Investigación Clínica de Navarra. Para asegurar el anonimato de los participantes, cada cuaderno de recogida de datos fue identificado con un código numérico.

El análisis estadístico se realizó con el programa IBM SPSS Statistics 21 realizándose análisis descriptivos para las variables cualitativas y cuantitativas (frecuencias, porcentajes, media y desviación típica); análisis exploratorio del IBDQ total y por dimensiones; pruebas de contraste de hipótesis (Kruskal-Wallis, U de MannWhitney), previo análisis de la distribución de los datos (Kolmogorov-Smirnow) y correlación de Spearman.

\section{RESULTADOS}

Las características sociodemográficas y clínicas de la población de estudio se muestran en la tabla 1.

La media de la puntuación total del cuestionario IBDQ es de 166 puntos (D.T.=40,06), con un rango de 41-219. En la dimensión digestiva, la media es 52,01 (D.T. $=13,51$; rango=13-70); 24,28 (D.T. $=7,29$; rango $=5-35)$ en la dimensión sistémica; 62,18 (D.T. $=14,01$; rango=18-83) para la dimensión emocional; finalmente la dimensión social presenta una media de 27,53 (D.T.=8,05) y un rango de 5-35. Hubo una pérdida de valores en un $7 \%$ de la muestra.

Las tablas 2 y 3 muestran los resultados al comparar la puntuación total y por dimensiones del IBDQ respecto a las variables sociodemográficas y clínicas. Se han hallado diferencias estadísticamente significativas para la puntuación total del IBDQ32 en función del sexo $(\mathrm{p}=0,001)$ y del tipo de enfermedad $(\mathrm{p}=0,005)$.

Como se puede apreciar en la tabla 2 , la media de la puntuación total del IBDQ es 180,59 (D.T. $=31,39$ ) en hombres y 152,45 (D. $T .=43,27$ ) en mujeres, siendo la diferencia estadísticamente significativa con un valor de $\mathrm{p}=0,01$. Estas diferencias entre sexo con significación estadística se produce también en las medias de la dimensión digestiva, sistémica, y emocional. 
Tabla 1. Características de la muestra

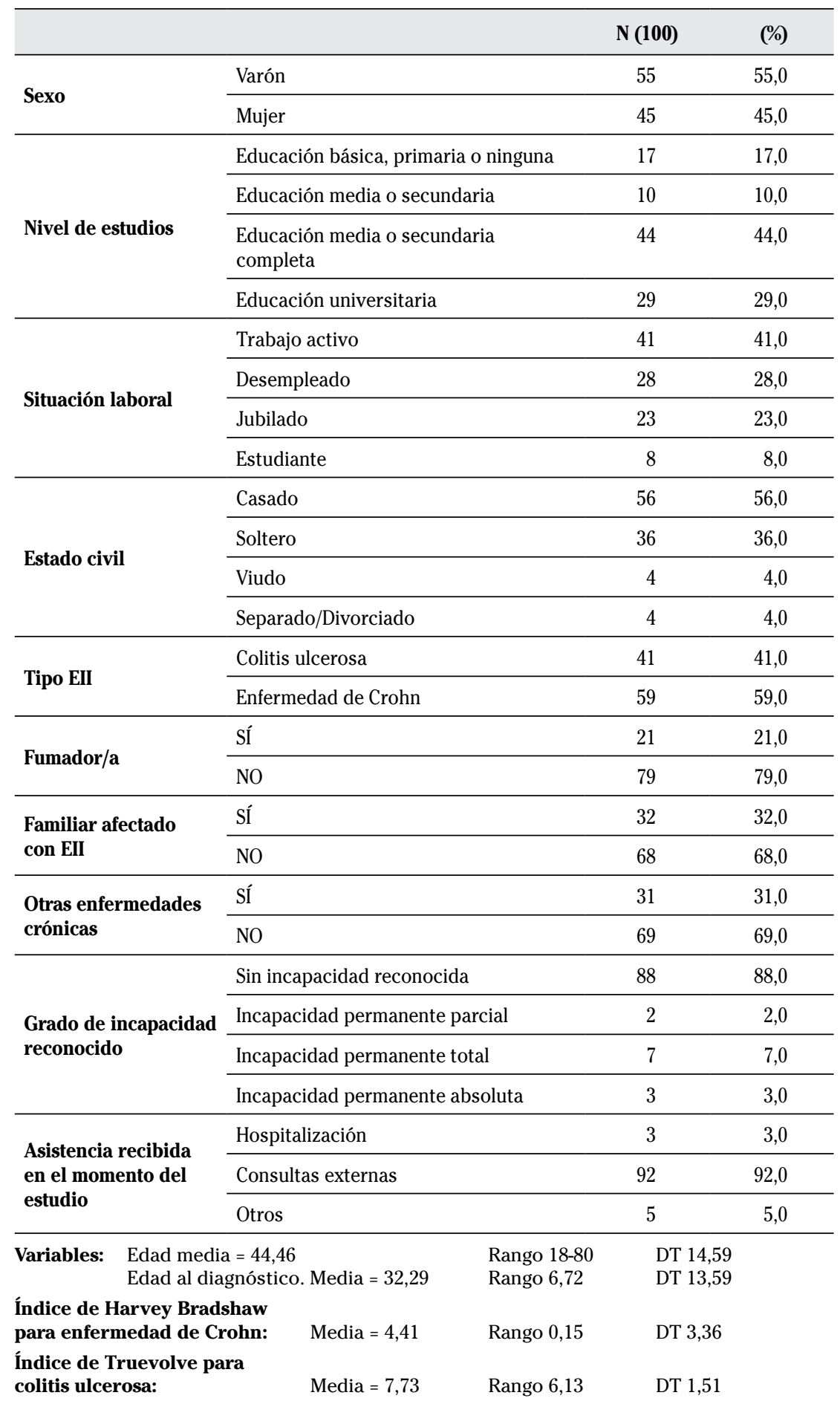


Tabla 2. Comparación IBDQ-32 con variables sociodemográficas y clínicas

\begin{tabular}{|c|c|c|c|c|c|c|}
\hline \multirow[b]{2}{*}{ Variable } & \multirow[b]{2}{*}{$\mathbf{n}$} & \multicolumn{5}{|c|}{ IBDQ-32 } \\
\hline & & $\begin{array}{c}\text { Digestivo } \\
\text { Media (D.T.) }\end{array}$ & $\begin{array}{c}\text { Sistémica } \\
\text { Media (D.T.) }\end{array}$ & $\begin{array}{l}\text { Emocional } \\
\text { Media (D.T.) }\end{array}$ & $\begin{array}{c}\text { Social } \\
\text { Media (D.T.) }\end{array}$ & $\begin{array}{c}\text { Total } \\
\text { Media } \\
\text { (D.T.) }\end{array}$ \\
\hline \multicolumn{7}{|l|}{ Sexo } \\
\hline Varón & 45 & $\begin{array}{c}56,46 \\
(12,13) \\
\end{array}$ & $\begin{array}{l}27,17 \\
(5,97)\end{array}$ & $\begin{array}{c}66,68 \\
(10,55) \\
\end{array}$ & $\begin{array}{l}30,27 \\
(5,70)\end{array}$ & $\begin{array}{l}180,59 \\
(31,39)\end{array}$ \\
\hline Mujer & 48 & $\begin{array}{c}47,77 \\
(13,80) \\
\end{array}$ & $\begin{array}{l}21,77 \\
(7,71)\end{array}$ & $\begin{array}{c}58,06 \\
(15,44) \\
\end{array}$ & $\begin{array}{l}24,85 \\
(9,16)\end{array}$ & $\begin{array}{l}152,45 \\
(43,27)\end{array}$ \\
\hline $\mathbf{p}$ & 93 & $<0,001$ & 0,001 & 0,004 & 0,012 & 0,001 \\
\hline \multicolumn{7}{|l|}{ Nivel de estudios } \\
\hline Educación básica o ninguna & 17 & $\begin{array}{c}49,12 \\
(13,68) \\
\end{array}$ & $\begin{array}{l}21,00 \\
(7,41)\end{array}$ & $\begin{array}{c}56,35 \\
(16,25) \\
\end{array}$ & $\begin{array}{l}25,53 \\
(8,88) \\
\end{array}$ & $\begin{array}{l}152,00 \\
(42,99)\end{array}$ \\
\hline Educación media & 10 & $\begin{array}{c}50,88 \\
(14,73) \\
\end{array}$ & $\begin{array}{l}25,00 \\
(6,67)\end{array}$ & $\begin{array}{c}63,00 \\
(17,31) \\
\end{array}$ & $\begin{array}{l}29,13 \\
(8,64)\end{array}$ & $\begin{array}{l}168,00 \\
(42,14)\end{array}$ \\
\hline $\begin{array}{l}\text { Educación secundaria } \\
\text { completa }\end{array}$ & 38 & $\begin{array}{c}53,06 \\
(15,57) \\
\end{array}$ & $\begin{array}{l}25,67 \\
(8,02) \\
\end{array}$ & $\begin{array}{c}64,31 \\
(14,10) \\
\end{array}$ & $\begin{array}{l}27,78 \\
(8,05)\end{array}$ & $\begin{array}{l}170,81 \\
(43,68) \\
\end{array}$ \\
\hline Educación universitaria & 28 & $\begin{array}{c}52,15 \\
(10,95)\end{array}$ & $\begin{array}{l}24,30 \\
(6,50)\end{array}$ & $\begin{array}{c}62,44 \\
(10,75)\end{array}$ & $\begin{array}{l}27,48 \\
(8,00)\end{array}$ & $\begin{array}{l}166,37 \\
(33,93)\end{array}$ \\
\hline p & 93 & 0,410 & 0,133 & 0,355 & 0,607 & 0,243 \\
\hline \multicolumn{7}{|l|}{ Situación laboral } \\
\hline Trabaja & 63 & $\begin{array}{c}51,69 \\
(13,58) \\
\end{array}$ & $\begin{array}{l}24,31 \\
(7,35)\end{array}$ & $\begin{array}{c}62,25 \\
(13,33) \\
\end{array}$ & $\begin{array}{l}26,98 \\
(8,30) \\
\end{array}$ & $\begin{array}{l}165,23 \\
(40,02)\end{array}$ \\
\hline Desempleado/a & 15 & $\begin{array}{c}56,07 \\
(11,19) \\
\end{array}$ & $\begin{array}{l}25,00 \\
(6,48) \\
\end{array}$ & $\begin{array}{c}64,07 \\
(13,85) \\
\end{array}$ & $\begin{array}{l}30,21 \\
(6,65) \\
\end{array}$ & $\begin{array}{r}175,36 \\
(33,01) \\
\end{array}$ \\
\hline Jubilado/a & 9 & $\begin{array}{c}48,44 \\
(17,77) \\
\end{array}$ & $\begin{array}{l}23,56 \\
(9,68) \\
\end{array}$ & $\begin{array}{c}60,00 \\
(18,54) \\
\end{array}$ & $\begin{array}{l}27,22 \\
(9,74) \\
\end{array}$ & $\begin{array}{l}159,22 \\
(54,50)\end{array}$ \\
\hline Estudiante & 6 & $\begin{array}{c}46,50 \\
(14,38) \\
\end{array}$ & $\begin{array}{l}23,00 \\
(9,05) \\
\end{array}$ & $\begin{array}{c}57,25 \\
(17,68) \\
\end{array}$ & $\begin{array}{l}23,75 \\
(7,22)\end{array}$ & $\begin{array}{r}150,50 \\
(45,68) \\
\end{array}$ \\
\hline p & 93 & 0,786 & 0,994 & 0,912 & 0,503 & 0,854 \\
\hline \multicolumn{7}{|l|}{ Estado civil } \\
\hline Casado/a & 55 & $\begin{array}{c}51,79 \\
(13,98) \\
\end{array}$ & $\begin{array}{l}23,64 \\
(7,70)\end{array}$ & $\begin{array}{c}61,94 \\
(14,49) \\
\end{array}$ & $\begin{array}{l}27,04 \\
(8,89) \\
\end{array}$ & $\begin{array}{l}164,42 \\
(42,86)\end{array}$ \\
\hline Soltero/a & 33 & $\begin{array}{c}51,87 \\
(13,22) \\
\end{array}$ & $\begin{array}{l}24,90 \\
(6,73)\end{array}$ & $\begin{array}{c}61,68 \\
(13,64)\end{array}$ & $\begin{array}{l}27,65 \\
(6,86)\end{array}$ & $\begin{array}{l}166,10 \\
(36,98)\end{array}$ \\
\hline Viudo/a & 2 & $\begin{array}{l}60,50 \\
(9,19)\end{array}$ & $\begin{array}{l}31,50 \\
(, 707) \\
\end{array}$ & $\begin{array}{l}65,50 \\
(7,77) \\
\end{array}$ & $\mathrm{n} / \mathrm{a}^{*}$ & $\begin{array}{l}191,50 \\
(2,12)\end{array}$ \\
\hline Separado/a-Divorciado/a & 2 & $\begin{array}{c}43,00 \\
(22,62) \\
\end{array}$ & $\begin{array}{c}24,50 \\
(14,84) \\
\end{array}$ & $\begin{array}{c}68,50 \\
(19,09) \\
\end{array}$ & $\begin{array}{c}25,50 \\
(13,43)\end{array}$ & $\begin{array}{l}161,50 \\
(70,00)\end{array}$ \\
\hline p & 92 & 0,744 & 0,479 & 0,895 & 0,901 & 0,924 \\
\hline \multicolumn{7}{|l|}{ Tipo de enfermedad } \\
\hline $\mathrm{CU}$ & 37 & $\begin{array}{c}55,47 \\
(11,34) \\
\end{array}$ & $\begin{array}{l}27,22 \\
(6,01)\end{array}$ & $\begin{array}{c}67,06 \\
(10,91) \\
\end{array}$ & $\begin{array}{l}30,50 \\
(6,88) \\
\end{array}$ & $\begin{array}{l}180,25 \\
(32,35)\end{array}$ \\
\hline $\mathrm{EC}$ & 54 & $\begin{array}{c}49,29 \\
(14,68)\end{array}$ & $\begin{array}{l}22,25 \\
(7,68)\end{array}$ & $\begin{array}{c}58,63 \\
(14,93)\end{array}$ & $\begin{array}{l}25,21 \\
(8,35)\end{array}$ & $\begin{array}{l}155,38 \\
(42,73)\end{array}$ \\
\hline
\end{tabular}




\begin{tabular}{|c|c|c|c|c|c|c|}
\hline \multirow[b]{2}{*}{ Variable } & \multirow[b]{2}{*}{$\mathbf{n}$} & \multicolumn{5}{|c|}{ IBDQ-32 } \\
\hline & & $\begin{array}{l}\text { Digestivo } \\
\text { Media (D.T.) }\end{array}$ & $\begin{array}{c}\text { Sistémica } \\
\text { Media (D.T.) }\end{array}$ & $\begin{array}{l}\text { Emocional } \\
\text { Media (D.T.) }\end{array}$ & $\begin{array}{c}\text { Social } \\
\text { Media (D.T.) }\end{array}$ & $\begin{array}{c}\text { Total } \\
\text { Media } \\
\text { (D.T.) }\end{array}$ \\
\hline $\mathbf{p}$ & 91 & 0,066 & 0,002 & 0,010 & 0,001 & 0,005 \\
\hline \multicolumn{7}{|l|}{ Fumador/a } \\
\hline Sí & 21 & $\begin{array}{c}51,50 \\
(12,72) \\
\end{array}$ & $\begin{array}{l}22,61 \\
(8,10) \\
\end{array}$ & $\begin{array}{c}59,94 \\
(14,30) \\
\end{array}$ & $\begin{array}{l}26,83 \\
(7,35) \\
\end{array}$ & $\begin{array}{l}160,89 \\
(38,68) \\
\end{array}$ \\
\hline No & 71 & $\begin{array}{c}51,90 \\
(14,02)\end{array}$ & $\begin{array}{l}24,71 \\
(7,24)\end{array}$ & $\begin{array}{c}62,63 \\
(13,97)\end{array}$ & $\begin{array}{l}27,51 \\
(8,41)\end{array}$ & $\begin{array}{l}166,76 \\
(41,17)\end{array}$ \\
\hline $\mathbf{p}$ & 92 & 0,586 & 0,235 & 0,376 & 0,450 & 0,382 \\
\hline \multicolumn{7}{|c|}{ Familiar afectado con EII } \\
\hline Sí & 11 & $\begin{array}{c}53,40 \\
(14,80)\end{array}$ & $\begin{array}{l}24,90 \\
(7,40)\end{array}$ & $\begin{array}{c}65,30 \\
(15,20)\end{array}$ & $\begin{array}{l}29,80 \\
(6,89)\end{array}$ & $\begin{array}{l}173,40 \\
(42,47)\end{array}$ \\
\hline No & 82 & $\begin{array}{c}51,62 \\
(13,63) \\
\end{array}$ & $\begin{array}{l}24,21 \\
(7,47) \\
\end{array}$ & $\begin{array}{c}61,67 \\
(13,88)\end{array}$ & $\begin{array}{l}27,06 \\
(8,30)\end{array}$ & $\begin{array}{l}164,55 \\
(40,45)\end{array}$ \\
\hline $\mathbf{p}$ & 93 & 0,309 & 0,555 & 0,141 & 0,235 & 0,203 \\
\hline \multicolumn{7}{|c|}{ Otras enfermedades crónicas } \\
\hline Sí & 24 & $\begin{array}{c}50,21 \\
(17,06) \\
\end{array}$ & $\begin{array}{l}23,33 \\
(9,77) \\
\end{array}$ & $\begin{array}{c}60,96 \\
(17,00) \\
\end{array}$ & $\begin{array}{l}26,46 \\
(9,76) \\
\end{array}$ & $\begin{array}{l}160,96 \\
(51,84) \\
\end{array}$ \\
\hline No & 69 & $\begin{array}{c}52,42 \\
(12,30) \\
\end{array}$ & $\begin{array}{l}24,64 \\
(6,39) \\
\end{array}$ & $\begin{array}{c}62,50 \\
(12,82) \\
\end{array}$ & $\begin{array}{l}27,72 \\
(7,54) \\
\end{array}$ & $\begin{array}{l}167,28 \\
(35,72) \\
\end{array}$ \\
\hline $\mathbf{p}$ & 93 & 0,861 & 0,993 & 0,923 & 0,621 & 0,968 \\
\hline
\end{tabular}

* Media IBDQ-32 Dimensión social es una constante cuando estado civil = Viudo y se ha desestimado.

En lo que respecta al tipo de enfermedad, los sujetos con EC presentan una media en la puntuación total del IBDQ de 180,25 (D.T. 32,25 ), mientras que los que padece CU la puntuación es de 155,38 (D.T.=42,73). La diferencia estadísticamente significativa se recoge también en la dimensión sistémica y en la social (Tabla 3).

Tabla 3. Correlación IBDQ- 32 con edad y edad al diagnóstico

\begin{tabular}{lccccc}
\hline \multirow{2}{*}{ Variable } & \multicolumn{5}{c}{ IBDQ-32 } \\
\cline { 2 - 6 } & Digestivo & Sistémica & Emocional & Social & Total \\
\hline Coeficiente de correlación & 0,011 & $-0,025$ & $-0,077$ & $-0,039$ & $-0,032$ \\
\hline Significación (bilateral) & 0,914 & 0,809 & 0,463 & 0,714 & 0,757 \\
\hline $\mathrm{N}$ & 93 & 93 & 93 & 93 & 93 \\
\hline Edad al diagnóstico & & & & & \\
\hline Coeficiente de correlación & $-0,094$ & $-0,103$ & $-0,115$ & $-0,117$ & $-0,114$ \\
\hline Significación (bilateral) & 0,370 & 0,326 & 0,271 & 0,265 & 0,277 \\
\hline $\mathrm{N}$ & 93 & 93 & 93 & 93 & 93 \\
\hline
\end{tabular}




\section{DISCUSIÓN}

Uno de los datos más relevantes obtenidos en este estudio es la influencia del sexo en la CVRS en este tipo de pacientes. Tal y como muestran los datos, existe una diferencia en las puntuaciones medias que son significativas. En el caso de la puntuación total del IBDQ-32 las mujeres presentan una media de 152,45 puntos con respecto a la de los hombres que es de 180,59, siendo la diferencia de 28,14 puntos. Los autores del IBDQ- $32^{4}$ en un análisis desagregado de las puntuaciones muestran que se considera una baja calidad de vida cuando la puntuación total de IBDQ-32 oscila entre 32 a 95, moderada entre 96 a 159 y alta calidad entre 160 a 224 puntos. Por tanto, los hombres estarían en un rango de alta calidad mientras que las mujeres se encontrarían con una calidad de vida moderada.

En línea con este hallazgo, se encuentran estudios que corroboran la influencia del sexo en relación a la percepción de la CVRS en pacientes con EII. Concluyendo que el sexo resulta ser un factor que influye en el deterioro de la CVRS en este tipo de pacientes, siendo las mujeres las que presentan una peor valoración de la CVRS con respecto a los hombres ${ }^{3,8}$, aspecto que se acentúa si la mujer es joven y si su patología es una enfermedad de Crohn'.

En relación a la identificación de la variable tipo de EII (EC o CU) como factor predictor de una mejor o peor CVRS, hay que señalar que en este estudio se ha observado una diferencia significativa que muestra datos más negativos en los pacientes con EC. En el caso de la puntuación total del IBDQ-32, los pacientes con enfermedad de Crohn presentan un media de 155,38 puntos con respecto a las personas con colitis ulcerosa que es de 180,25 , siendo la diferencia de 24,87 puntos. Estas diferencias muestran que con la EC se vive con una calidad de vida moderada con respecto a la alta calidad de vida que perciben las personas con CU.

La evidencia científica consultada es heterogénea en este sentido. Se han identificado algunos estudios que comparten estos resultados y han mostrado que los pacientes con EC valoran peor CVRS en comparación con los pacientes con $\mathrm{CU}^{8,10}$. Por otra parte, también existen otros estudios donde no se han mostrado diferencias ni existencia de correlación significativa entre el grupo estudiado según el tipo de la enfermedad con respecto a la calidad de vida $^{11,12}$.

Estos resultados, contradictorios a priori, podrían justificarse si se considera que los sujetos con EC, a diferencia de los que padecen $\mathrm{CU}$, podrían ser más propensos a experimentar de forma coadyuvada una mayor presencia de ansiedad que repercutiría en una peor CVRS ${ }^{13}$. No obstante, la hipótesis de que la CVRS varía entre los pacientes con EII con diagnósticos diferentes presenta una disyuntiva, a la luz de los resultados de esta y otras investigaciones, que requiere de más estudios que profundicen y clarifiquen estos resultados con una muestra más elevada, que permita ofrecer una evidencia más sólida, así como con estudios que incluyan correlaciones con otros factores diferenciadores entre EC y CU.

Otro hallazgo de interés es que la mayoría de sujetos estudiados no tienen ninguna incapacidad reconocida oficialmente; tan sólo un porcentaje mínimo declaran una incapacidad permanente parcial, total o absoluta. Hay que hacer constar que en nuestro estudio la puntuación de los índices de gravedad de la enfermedad sitúan a la muestra en un nivel "activa-leve" para los sujetos con CU y "en remisión" para los sujetos con EC. Tal y como corroboran otros trabajos, las EII no son consideradas enfermedades incapacitantes, en comparación con otros enfermedades inflamatorias crónicas tales como la esclerosis múltiple ${ }^{14}$. La CVRS es un indicador de discapacidad poco reconocido, pero que a la luz de los resultados de este trabajo, sí que apunta a una tendencia incapacitante en pacientes con EII, especialmente si el tipo de enfermedad es EC o la padecen mujeres. No obstante, resulta necesario investigación adicional para cuantificar mejor la discapacidad en pacientes con EII ${ }^{15}$, estableciendo corres- 
pondencias entre distintos parámetros de la enfermedad, entre ellos la afectación de la CVRS, y los grados de discapacidad e incapacidad reconocidos oficialmente en nuestro país.

Este estudio presenta algunas limitaciones. La recogida de datos se ha hecho mediante entrevista personal en el momento de la consulta en el servicio de digestivo, lo cual ha dificultado esta parte del estudio, de ahí que el número de sujetos incluidos no haya podido ser mayor. Hay ausencia de datos en algunas de las variables, que corresponde en su totalidad a errores en la tabulación de los mismos y que no pudieron ser subsanados. No obstante, el porcentaje de valores perdidos ha sido como máximo de un $9 \%$, lo cual no es un valor representativo que pudiera invalidar los resultados del trabajo. Otra limitación consiste en que el $92 \%$ de los pacientes estaban recibiendo atención sanitaria de forma extrahospitalaria, pudiendo existir diferencias en términos de CVRS entre este tipo de pacientes y los hospitalizados. No obstante, el criterio diferencial a tener en cuenta no es tanto la asistencia recibida sino la situación de actividad o remisión de la enfermedad. Por último, sería conveniente ampliar muestra para verificar algunas de las asociaciones estadísticamente significativas que se han hallado. Desde los servicios de salud se debe incidir en que la gestión de los pacientes con EII no contemple solo el control de los síntomas y la curación de las lesiones, sino también la normalización de su calidad de vida. Por ello, el conocimiento de factores predictores y su influencia puede ser utilizado en el diseño e implementación de intervenciones en salud específicas dirigidas hacia aquellos pacientes más vulnerables en términos de riesgo de pérdida de CVRS.

\section{Agradecimientos}

$\mathrm{Al}$ equipo médico del Servicio Digestivo del Complejo Hospitalario de Navarra; a Dña. Ana César, jefe de área de enfermería del Centro Príncipe de Viana; a Dña. Rosana de la Rosa, estudiante de doctorado en el Departamento de Ciencias de la Salud de la Universidad Pública de Navarra. A todos los pacientes que han participado en el estudio.

\section{BIBLIOGRAFÍA}

1. Van Assche G, Dignass A, Panes J, Beaugerie L, Karagiannis J, Allez M et al. European Crohn's and Colitis Organisation (ECCO). The second European evidence-based Consensus on the diagnosis and management of Crohn's disease: Definitions and diagnosis. J Crohn's colitis 2010; 4: 7-27.

2. Masachs M, Casellas F, Malagelada J. Traducción, adaptación y validación al español del cuestionario de calidad de vida de 32 ítems (IBDQ-32) de la enfermedad inflamatoria intestinal. Rev Esp Enferm Dig 2007; 99: 511519.

3. GiLı M, BAuŻ̀ N. Calidad de vida y personalidad en la enfermedad inflamatoria intestinal. Gastroenterol Hepatol 2009; 32 (Suppl 2): 3-8.

4. Guyatt G, Mitchell A, Irvine EJ, Singer J, WiLLIAMS N, GoODACRE R et al. A new measure of health status for clinical trials in inflammatory bowel disease. Gastroenterology 1989; 96: 804-810.

5. Pallis AG, Mouzas IA, VlachoniKolis IG. The inflammatory bowel disease questionnaire: a review of its national validation studies. Inflamm Bowel Dis 2004; 10: 261-269.

6. HaApamäki J, Roine R, Sintonen H, Turunen U, FARKKILA M, PERTTU E et al. Health-related quality of life in inflammatory bowel disease measured with the generic 15D instrument. Qual Life Res 2010; 19: 919-928.

7. Vidal Á, Gómez-Gil E, Sans M, Portella MJ, SALAmero M, Piqué JM et al. Psychometric properties of the original Inflammatory Bowel Disease Questionnaire, a Spanish version. Gastroenterol Hepatol 2007; 30: 212-218.

8. Bernklev T, Jahnsen J, Aadland E, Sauar J, Schulz T, Lygren I et al. Health-related quality of life in patients with inflammatory bowel disease five years after the initial diagnosis. Scand J Gastroenterol 2004; 39: 365-373.

9. Saibeni S, Cortinovis I, Beretta L, Tatarella M, Ferraris L, RondonotTi E et al. Gender and disease activity influence health-related quality of life in inflammatory bowel diseases. Hepato-Gastroenterol 2005; 52: 509-515.

10. Larsson K, LöÖF L, Rönnblom A, Nordin K. Quality of life for patients with exacerbation in 
inflammatory bowel disease and how they cope with disease activity. J Psychosom Res 2008; 64: 139-148.

11. Casellas F, Arenas JI, Baudet JS, Fábregas S, García $\mathrm{N}$, Gelabert $\mathrm{J}$ et al. Impairment of health-related quality of life in patients with inflammatory bowel disease: a Spanish multicenter study. Inflamm Bowel Dis 2005; 11 : 488-496.

12. Kalafateli M, Triantos C, Theocharis G, Giannakopoulou D, KoutroumpaKis E, Chronis A et al. Health-related quality of life in patients with inflammatory bowel disease: a single-center experience. Ann Gastroenterol 2013; 26: 243248.
13. Lix L, Graff L, Walker J, Clara I, Rawsthorne P, Rogala L et al. Longitudinal study of quality of life and psychological functioning for active, fluctuating, and inactive disease patterns in inflammatory bowel disease. Inflamm Bowel Dis 2008; 14: 1575-1584.

14. Peyrin-Biroulet L, Cieza A, Sandborn Wu, Coenen M, Chowers Y, Hibi T et al. Development of the first disability index for inflammatory bowel disease based on the international classification of functioning, disability and health. Gut 2012; 61: 241-247.

15. Büsch K, Sonnenberg A, Bansback N. Impact of inflammatory bowel disease on disability. Curr Gastroenterol Rep 2014; 16: 414. doi: 10.1007/s11894-014-0414-0 
